\title{
Development of an ATP and hydrogen ion image sensor using a patterned Apyrase-immobilized membrane
}

\author{
You-Na Lee ${ }^{a^{*}}$, Koichi Okumura, ${ }^{\mathrm{a}, \mathrm{b}}$, Tatsuya Iwata ${ }^{\mathrm{a}}$, Kazuhiro Takahashi ${ }^{\mathrm{a}}$, Toshiaki Hattori ${ }^{\mathrm{a}}$, Makoto \\ Ishida $^{\text {a,b }}$, Kazuaki Sawada ${ }^{\text {a,b }}$ \\ ${ }^{a}$ Electrical \& Electronic Information Eng., Hibarigaoka 1-1, Tempaku-cho, Toyohashi University of \\ Technology, Toyohashi, Japan 441-8580; \\ ${ }^{\mathrm{b}}$ Electronics-Inspired Interdisciplinary Research Institute, Hibarigaoka 1-1, Tempaku-cho, Toyohashi \\ University of Technology, Toyohashi, Japan 441-8580 \\ *Author for correspondence: lee-y@int.ee.tut.ac.jp
}

\begin{abstract}
A bio-image sensor using a patterned apyrase-immobilized membrane was developed to visualize the activities of adenosine triphosphate (ATP) and $\mathrm{H}^{+}$ion in real-time. An enzymatic membrane patterning technique was suggested to immobilize apyrase on a specific sensing area of a charge coupled device (CCD)-type image sensor. It was able to observe the spatiotemporal information of ATP and $\mathrm{H}^{+}$ion. The smallest size of a patterned membrane is $250 \times 250 \mu \mathrm{m}^{2}$. The fabrication parameters of the patterned membrane, such as its thickness and the intensity of the incident light used for photolithography, were optimized experimentally. The sensing area under the patterned apyrase-immobilized membrane revealed a linear response up to $0.6 \mathrm{mM}$ of ATP concentration with a sensitivity of $37.8 \mathrm{mV} / \mathrm{mM}$. Meanwhile, another sensing area without the patterned membrane measured the diffused $\mathrm{H}^{+}$ion from nearby membranes. This diffusion characteristics were analyzed to determine a measurement time that can minimize the undesirable impact of the diffused ions. In addition, the newly developed bio-image sensor successfully reconstructed ATP and $\mathrm{H}^{+}$ion dynamics into sequential 2-dimensional images.
\end{abstract}

\section{Keywords}

Bio-image sensor, Enzyme-immobilized membrane, Apyrase, ATP, $\mathrm{H}^{+}$ion 


\section{Introduction}

Adenosine triphosphate (ATP) is a well-known essential energy source for almost all cellular metabolisms. In addition, it also can regulate many biological processes in the central nervous system as a neurotransmitter. ATP is released separately or simultaneously with other neurotransmitters, such as acetylcholine, noradrenaline, and dopamine, to mediate neuronal signaling and the development of central synapses [1-3]. In addition, recent studies indicate that the change of extracellular ATP levels can be used to diagnose hypertension, cancer cells and Alzheimer's disease [4-6]. Thus, it is very important to identify the levels of ATP concentration in an extracellular environment. Several analytical methods, including enzymatic biosensors based on an ion selective field effect transistor [7] and a metal microelectrode [8,9], have long been the preferred method to detect ATP, because of its structural advantage and excellent temporal resolution. However, these methods are incapable of providing the spatial resolution for measuring the activity of neurotransmitters in the central nervous system. In an attempt to localize extracellular ATP release with high spatial resolution, scanning electrochemical microscopy has been described in literature as a model for in vitro monitoring of the surface condition of bone cell $[10,11]$. Beside those methods, indirect optical detection using fluorescent and chemiluminescent activities have been successfully demonstrated for a high sensitive determination of ATP concentration [12-16]. However, they require difficult nervous tissue sampling or preprocessing, like the fluorescent labeling of biomolecules. To provide an advanced analytical method which can include the advantages of the conventional methods, we have attempted to develop a bio-image sensor based on a charge-coupled device (CCD)-type image sensor $[17,18]$.

In this article, we propose and demonstrate a multi-ion image sensor consisting of a patterned apyrase-immobilized membrane on a CCD-type image sensor to determine simultaneously ATP and $\mathrm{H}^{+}$ ion activities. ATP concentration is determined by measuring the local $\mathrm{pH}$ change from an enzymatic reaction that leads to the formation of $\mathrm{H}^{+}$ions. However, when using this method it is difficult to distinguish between the produced $\mathrm{H}^{+}$ions and the other $\mathrm{H}^{+}$ions that are diffused from nearby membranes or nerve samples [19]. To overcome this limitation, a cross-linkable polymer [20] was used as the most suitable material among various immobilization materials [21-23], because it is able to immobilize enzymes and be patterned on a selective area of the CCD-type image sensor by a conventional photolithographic method, which is compatible with the complementary metal-oxide semiconductor (CMOS) process. The fabrication parameters of the membrane, such as its thickness and the intensity of the incident light for photolithography, are experimentally optimized without any loss of enzymatic activity. While the sensing area under the patterned membranes is detecting ATP activities spatiotemporally, the other sensing area without the membrane monitors the diffused $\mathrm{H}^{+}$ions as a $\mathrm{pH}$ sensor. Those measured results are successfully reconstructed in 2-dimensions and used to investigate the diffusion property of the $\mathrm{H}^{+}$ions between the patterns. In the future, we expect that the proposed ATP and $\mathrm{H}^{+}$ion image sensor can be applied to analyze ATP levels in the central nervous system or a cell culture 
medium. Moreover, the sensor is thought to investigate the direct vasodilator actions of hydrogen sulfide mediated by ATP-sensitive $\mathrm{K}^{+}$channels to regulate blood pressure in the hypothalamus [24].

\section{Experiments}

2.1. Materials

ATP disodium salt $n$-hydrate, $\mathrm{MgCl}_{2}(1 \mathrm{M})$ and apyrase (EC 3.6.1.5) were purchased from Wako Pure Chemicals (Osaka, Japan), Nippon Gene Co. (Tokyo, Japan) and Sigma-Aldrich Co. (St. Louis, MO, USA), respectively. Apyrase was mixed with BIOSIRFINE ${ }^{\circledR}$-AWP from Toyo Cosei Co. (Tokyo, Japan) for crosslinking. Sodium salt and HEPES were purchased from Dojindo Laboratories (Kumamoto, Japan). ATP and buffer solution were brought to $\mathrm{pH} 8.0$ with sodium hydroxide at room temperature $\left(25 \pm 2^{\circ} \mathrm{C}\right)$, because the degradation process of ATP is active below $\mathrm{pH} 8.0$ [25]. And HEPES concentration in the solutions was $1 \mathrm{mM}$. To prepare ATP solutions, ATP disodium salt $n$-hydrate had dissolved in buffer solution with various concentrations from $50 \mathrm{mM}$ to $0.01 \mathrm{mM}$. In experiments, these solutions were diluted with the buffer solution in a 1:9 ratio for the analysis of the ATP reaction. As the same way, $\mathrm{pH} 4.1$ buffer solution was tested to measure the $\mathrm{H}^{+}$concentration change from $\mathrm{pH} 8.0$ to $\mathrm{pH}$ 7.1.

\subsection{Preparation of the apyrase-immobilized membrane}

The apyrase-immobilized membrane was deposited as described in figure 1 (a). A cross-linkable polymer was used to immobilize apyrase in the membrane on a CCD-type image sensor that was rinsed in distilled water at $80^{\circ} \mathrm{C}$ for $30 \mathrm{~min}$. Apyrase and cross-linked polymer were then mixed at a 1:4 ratio. The mixture was immediately spread on top of the image sensor, and exposed to UV light of a mask aligner (USH-500BY1; Ushio Inc., Tokyo, Japan) for cross-linking. Before measurements with ATP solution, all samples were rinsed with distilled water to remove the uncured polymer. Figure 1 (b) shows an optical image of the surface of the completed bio-image sensor. The attempted smallest size of the pattern was $250 \times 250 \mu \mathrm{m}^{2}$. The experimented parameters to optimize the membrane, such as its thickness and the incident of light power source for photolithography, will be discussed.

\section{Results and discussion}

\subsection{Measurement principle of the bio-image sensor}

The proposed ATP and $\mathrm{H}^{+}$ion image sensor is based on a CCD-type image sensor that was developed by our group. The sensor has a resolution of $128 \times 128$ pixels. Each pixel can detect the varying $\mathrm{H}^{+}$ concentration with the $\mathrm{pH}$ sensitivity of $35.8 \mathrm{mV} / \mathrm{pH}$ as reported in our previous report [26]. The $\mathrm{pH}$ sensitivity of the sensor is not changed, even though the enzymatic membrane is deposited on the sensing area. However, the detection speed is just slightly delayed because the produced $\mathrm{H}^{+}$ions by the enzymatic reaction have to pass through the porous structure of the membrane. ATP concentrations can be determined by measuring the changed amount of $\mathrm{H}^{+}$ions after ATP degradation which was catalyzed by apyrase as follows [27]: 


$$
\begin{aligned}
& \text { ATP }+\mathrm{H}_{2} \mathrm{O} \stackrel{\text { Apyrase }}{\longrightarrow} \mathrm{ADP}+\mathrm{HPO}_{4}^{2-}+\mathrm{H}^{+} \\
& \mathrm{ADP}+\mathrm{H}_{2} \mathrm{O} \stackrel{\text { Apyrase }}{\longrightarrow} \mathrm{AMP}+\mathrm{HPO}_{4}^{2-}+\mathrm{H}^{+}
\end{aligned}
$$

When ATP solution ( $\mathrm{pH} \mathrm{8.0)} \mathrm{or} \mathrm{pH} 4.1$ buffer solution are dropped in the prepared $\mathrm{pH} 8.0$ buffer solution as shown in figure 2 (a) and (a'), the produced $\mathrm{H}^{+}$ions after dropping solutions change the potential level under the sensing area of a pixel. Figure 2 (b) and (b') shown that the changed value of the potential level is proportional to the amount of the increased $\mathrm{H}^{+}$ions. Then, the accumulated charge according to the potential level is read-out to $\mathrm{V}_{\text {OUT }}$ by the $\mathrm{CCD}$ technique. Finally, the measured signals from $128 \times 128$ pixels are reconstructed in real time into 2-dimensional images to provide the spatial and temporal information of the produced $\mathrm{H}^{+}$ions. The flame speed of the images is $30 \mathrm{frame} / \mathrm{s}$. The response is the average of the output voltage differences $\left(\Delta \mathrm{V}_{\text {OUT }}\right)$ of the measured pixels, which is defined as:

$$
\text { Response }=\frac{\sum_{j=1}^{n}\left(V_{j, \text { saturated }}-V_{j, \text { initial }}\right)}{n}
$$

where $n$ is numbers of the pixels, $V_{j, \text { initial }}$ is the initial output voltage and $V_{j, \text { saturated }}$ is the saturated output voltage that was measured at $160 \mathrm{~s}$ after the addition of the ATP.

\subsection{Bio-image sensor optimization}

To obtain an optimum bio-image sensor, the thickness of the membrane and the UV light power for photolithography were experimentally determined. Cross-linkable polymer containing apyrase was immobilized without patterns on the top of a CCD-type image sensor by a UV exposure of $1000 \mathrm{~mJ} / \mathrm{cm}^{2}$. The membrane thickness was controlled by the speed of a spin coater and measured by Microfigure measuring Instrument (ET 4000A; Kosaka Laboratory Ltd., Tokyo, Japan). In figure 3 (a), the membrane with a thickness of $0.3 \mu \mathrm{m}$ shows the highest response of $0.032 \mathrm{~V}$ for $1 \mathrm{mM}$ ATP solution. The decreased response means that the membrane does not contain enough apyrase to react with ATP when the thickness is thinner than $0.3 \mu \mathrm{m}$. Meanwhile, when the membrane is thicker than $0.3 \mu \mathrm{m}$, the movement of $\mathrm{H}^{+}$ions through the membrane is obstructed by the thick cross-linked polymer. Based on these results, an apyrase-immobilized membrane was patterned into a size of $250 \times 250 \mu^{2}$. UV light energy over 1000 $\mathrm{mJ} / \mathrm{cm}^{2}$ allowed us to obtain the saturated response to the ATP, as shown in figure 3 (b). The measured response corresponding to $1000 \mathrm{~mJ} / \mathrm{cm}^{2}$ was $0.025 \mathrm{~V}$ for $1-\mathrm{mM}$ ATP, and this light intensity is considered to be sufficient for patterning the membrane. From these results, the response of the sensor with the patterned membrane was lower than with the non-patterned membrane, even though the two types of membranes were deposited in the same conditions. That is the only difference between the two types, whether the apyrase-immobilized membrane is patterned or not. It indicates that the $\mathrm{H}^{+}$ion produced by 
enzymatic reactions can diffuse and be detected at adjacent pixels.

\subsection{ATP and $\mathrm{H}^{+}$ion sensing characterization}

Before ATP and $\mathrm{H}^{+}$ion sensing measurement, the optimized sensor was calibrated as shown in figure 4 (a). A linearity of calibration function was obtained in the concentration range up to $0.6 \mathrm{mM}$ ATP and the sensitivity was $37.8 \mathrm{mV} / \mathrm{mM}$ ATP with $\mathrm{R}^{2}=0.9934$. From this data, the limit of detection (LOD) of $1.3 \mu \mathrm{M}$ was calculated according to the definition of IUPAC [28]. Figure 4 (b) shows the repeatability error of the randomly selected 49 pixels including the membrane and their summary shows in table 1 . It means that the pixels of a bio-image sensor can perform equally in the total non-linearity error of $15.60 \%$. All output voltages of $128 \times 128$ pixels were recorded and reconstructed into 2 -dimensional images sequentially to provide visually intuitive interface. The output voltage difference was represented by a color. Figure 5 (a) shown that all pixels can detect $\mathrm{H}^{+}$concentration movement after dropping $\mathrm{pH} 4.1$ buffer solution into $\mathrm{pH}$ 8.0 buffer solution. The $\mathrm{pH}$ value of the mixed solution at $160 \mathrm{~s}$ was $\mathrm{pH} 7.1$. The detection delay at the sensing area of the patterned membranes was measured, because $\mathrm{H}^{+}$ions had to pass through the membrane. By the same sensor, the sequential images in figure 5 (b) were obtained after adding $10 \mathrm{mM}$ ATP solution droplet into $\mathrm{pH} 8.0$ buffer solution. The final ATP concentration in the mixed solution was 1 $\mathrm{mM}$. The real-time images are providing the spatiotemporal information of ATP movement, because the $\mathrm{pH}$ change was caused by the produced $\mathrm{H}^{+}$ions between ATP and the immobilized apyrase. However, the produced $\mathrm{H}^{+}$ions was able to diffuse through the solution and be detected by pixels without the apyrase-immobilized membrane. The gradually diffused $\mathrm{H}^{+}$ions were confirmed by the increased output voltage at $30 \mathrm{~s}$ and $160 \mathrm{~s}$. In order to obtain the measurement result without the effect of the diffused $\mathrm{H}^{+}$ ions, the measurement timing needs to be determined.

\subsection{Determination of the measuring time}

To determine the measuring time for the proposed sensor, the response data of 12 pixels from the 2-dimensional images of figure 5 (b) was extracted and used to evaluate the diffusion characteristics of $\mathrm{H}^{+}$ ions. Figure 6 shows the sequential response values corresponding to each pixels in an optical image, consisting of six pixels without the membrane in the center and the other six pixels with the apyrase-immobilized membrane on each side. Even though the six pixels without the membrane have no enzymatic reaction, the measured response at the area was gradually increased depending on the amount of the diffused $\mathrm{H}^{+}$ions from the adjacent pixels with the membrane. The biggest response difference between the two areas, where the membrane exits or the membrane does not exits, was observed at $10 \mathrm{~s}$ after adding ATP solution. When we consider the diffusion coefficient $\mathrm{D}$ of $\mathrm{H}^{+}$ions of $7.62 \times 10^{-5} \mathrm{~cm}^{2} / \mathrm{s}$ [29], it is assumed that the diffused $\mathrm{H}^{+}$ions at a patterned membrane takes approximately $10.3 \mathrm{~s}$ to reach to the nearest pixel of the next membrane. Hence, in order to minimize the effect of the diffused $\mathrm{H}^{+}$, it is appropriate that the activities of ATP should be measured within $10 \mathrm{~s}$ after adding ATP solution. 


\section{Conclusions}

In this study, a bio-image sensor based on a CCD-type image sensor was developed to observe $\mathrm{H}^{+}$ion and ATP dynamics simultaneously. An apyrase in a cross-linkable polymer was deposited on the sensing area of a CCD-type image sensor that can measure $\mathrm{pH}$ change. The apyrase-immobilized membrane was patterned by a photolithographic technique, and the fabrication parameters were experimentally optimized to maximize their response. The fabricated bio-image sensor with the patterned membrane was able to detect ATP and $\mathrm{H}^{+}$ions simultaneously and reconstructed the measured information into 2-dimensional images in real time. The measured spatiotemporal information was used to evaluate the diffusion property of $\mathrm{H}^{+}$ions among pixels. From these results, the measurement within $10 \mathrm{~s}$ after adding ATP solution was assumed to minimize the signal interference caused by diffused $\mathrm{H}^{+}$ions from the adjacent pixels. The suggested membrane patterning technique will be applied to develop a multiplexing bio-image sensor to determine various neurotransmitters simultaneously. 


\section{Acknowledgements}

This work was supported part by The Knowledge Hub of Aichi, The Priority Research Project from Aichi

Science \& Technology Foundation, JSPS KAKENHI Grant Number 24226010, and CREST, Japan

Science and Technology Agency.

\section{References}

[1] Y. Pankratov, U. Lalo, A. Verkhratsky, R.A. North, Vesicular release of ATP at central synapses, Euro. J. Phys. 452 (2006) 589-597.

[2] S. Koizumi, K. Fujishita, K. Inoue, Regulation of cell-to-cell communication mediated by astrocytic ATP in the CNS, Purin. Signal. 1 (2005) 211-217.

[3] E.K. Ainscow, S. Mirshamsi, T. Tang, M.L.J. Ashford, G.A. Rutter, Dynamic imaging of free cytosolic ATP concentration during fuel sensing by rat hypothalamic neurons: evidence for ATP-independent control of ATP-sensitive K ${ }^{+}$channels, J. Phys. 544 (2002) 429-445.

[4] Z. Guan, E.W. Inscho, Role of adenosine 5'-triphosphate in regulating renal microvascular function and in hypertension, Hypertension. 58 (2011) 333-340.

[5] I. Martins, Y. Wang, M. Michaud, Y. Ma, AQ Sukkurwala, S. Shen, O. Kepp, D. Métivier, L. Galluzzi, J-L Perfettini, L. Zitvogel and G. Kroemer, Molecular mechanisms of ATP secretion during immunogenic cell death, Cell Death Differ. 21 (2014) 79-91.

[6] M.J.P. de Lala and J. Pintor, Presence and release of ATP from the retina in an Alzheimer's disease model, J. Alzheimer's Dis. 43 (2015) 177-181.

[7] A. Kugimiya, and K. Kohara, Amino acid sensing using an ion-sensitive field-effect transistor, J. Chem, Chem. Eng. 6 (2012) 397-400.

[8] B.A. Patel, M. Rogers, T. Wieder, D. O’Hare, M.G. Boutelle, ATP microelectrode biosensor for stable long-term in vitro monitoring from gastrointestinal tissue, Biosen. Bioelectron. 26 (2011) 2890-2896.

[9] I.S. Kucherenko, D.Y. Kucherenko, O.O. Soldatkin, F. Lagarde, S.V. Dzyadevych, A.P. Soldatkin, A novel conductometric biosensor based on hexokinase for determination of adenosine triphosphate, Talanta 150 (2016) 469-475.

[10] E. Hecht, A. Liedert, A. Ignatius, B. Mizaikoff and C. Kranz, Local detection of mechanically induced ATP release from bone cells with ATP microbiosensors, Biosen. Bioelectron. 44 (2013) 27-33.

[11] A. Kueng, C. Kranz and B. Mizaikoff, Imaging of ATP membrane transport with dual micro-disk electrodes and scanning electrochemical microscopy, Biosen. Bioelectron. 21 (2005) 346-353.

[12] C. Wang, C.-Y.C. Huang, W.-C. Lin, Optical ATP biosensor for extracellular ATP measurement, Biosen. Bioelectron. 43 (2015) 355-361. 
[13] C. Lin, Y. Chen, Z. Cai, Z. Zhu, Y. Jiang, C.J. Yang, X. Chen, A label-free fluorescence strategy for sensitive detection of ATP based on the ligation-triggered super-sandwich, Biosens. Bioelectron. 63 (2015) $562-565$.

[14] K. Yamamoto, K. Furuya, M. Nakamura, E. Kobatake, M. Sokabe and J. Ando, Visualization of flow-induced ATP release and triggering of $\mathrm{Ca}^{+}{ }^{+}$waves at caveolae in vascular endothelial cells, J. Cell Sci. 124 (2011) 3477-3483.

[15] S. Bovetti, C. Moretti and T. Fellin, Mapping brain circuit function in vivo using two-photon fluorescence microscopy, Micro. Res. Tech. 77 (2014) 492-501.

[16] Z. Zhang, E. Sharon, R. Freeman, Z. Liu and I. Willner, Fluorescence detection of DNA, Adenosine-5'-Triphosphate (ATP), and Telomerase activity by Zinc( II )-Protoporphyrin IX/G-Quadruplex labels, Anal. Chem. 84 (2012) 4789-4797.

[17] M. Futagawa, D. Suzuki, R. Otake, F. Dasai, M. Ishida and K. Sawada, Fabrication of a $128 \times 128$ pixels charge transfer type hydrogen ion image sensor, IEEE Trans. Electron Devices 60 (2013) 2634-2639.

[18] H. Nakazawa, R. Otake, M. Futagawa, F. Dasai, M. Ishida and K. Sawada, High-sensitivity charge-transfer-type pH sensor with quasi-signal removal structure, IEEE Trans. Electron Devices 61 (2014) 136-140.

[19] T. Sakurai, A. Iwashita, K. Okumura, M. Ishida, and K. Sawada, Acetylcholine dynamics in cortical networks by an ion image sensor with neurotransmitter-sensitive magnetic nanomachines, Transducers (2013) 760-763.

[20] Y. Ito, M. Nagawa, M. Takeda and T. Shibuya, Photo-reactive polyvinylalcohol for photo-immobilized microarray, Biomaterials 26 (2005) 211-216.

[21] A. Sassolas, L.J. Blum and B.D. Leca-Bouvier, Immobilization strategies to develop enzymatic biosensors, Biotechnol. Advan. 30 (2012) 489-511.

[22] S. Schoffelen and J.C.M. van Hest, Multi-enzyme systems: bringing enzymes together in vitro, Soft Matter 8 (2012) 1736-1746.

[23] P. Jochems, Y. Satyawali, L. Diels and W. Dejonghe, Enzyme immobilization on/in polymeric membranes: status, challenges and perspectives in biocatalytic membrane reactors (BMRs), Green Chem. 13 (2011) 1609-1623.

[24] G.S. Dawe, S.P. Han, J.-S. Bian and P.K. Moore, Hydrogen sulphide in the hypothalamus causes an ATP-sensitive $\mathrm{K}+$ channel-dependent decrease in blood pressure in freely moving rats, Neuroscience 152 (2008) 169-177.

[25] S. Migita, K. Ozasa, T. Tanaka and T. Haruyama, Enzyme-based field-effect transistor for adenosine 
triphosphate (ATP) sensing, Analyt. Sci. 23 (2007) 45-48.

[26] P.L. Swart and C.K. Campbell, Gate-voltage limitations for thin channel MIS and Schottky buried-channel charge-coupled devices, Microelectronics J. 12 (1981) 5-10.

[27] Krishnan PS, Apyrase, pyrophosphatase and metaphosphatase of Penicillium chrysogenum, Arch. Biochem. Biophys. 37:1 (1952) 224-234.

[28] G.L. Long and J.D. Winefordner, Limit of detection a closer look at the IUPAT definition, Anal. Chem. $55: 7$ (1983) 712-724.

[29] S.H. Lee and J.C. Rasaiah, Proton transfer and the mobilities of the $\mathrm{H}+$ and $\mathrm{OH}-$ ions from studies of a dissociating model for water, J. Chem. Phys. 135 (2011) 124505. 
Table 1

Repeatability evaluation of different ATP concentrations.

\begin{tabular}{llll}
\hline $\begin{array}{l}\text { ATP concentration }(\mathrm{mM}), \\
\mathrm{n}=49\end{array}$ & $\begin{array}{l}\text { Average }(\mathrm{V}), \\
\mathrm{m}=(1 / \mathrm{n}) \sum \mathrm{x}_{\mathrm{i}}\end{array}$ & $\begin{array}{l}\text { Standard deviation }(\mathrm{V}), \\
\sigma=\sqrt{\left\{(1 / \mathrm{n}) \Sigma\left(\mathrm{x}_{\mathrm{i}}-\mathrm{m}\right)^{2}\right\}}\end{array}$ & $\begin{array}{l}\text { Coefficient of variation }(\%), \\
(\text { standard deviation/arithmetic average }) \times 100\end{array}$ \\
\hline Buffer & $1.53 \mathrm{E}-03$ & $6.82 \mathrm{E}-04$ & 44.66 \\
0.1 & $5.34 \mathrm{E}-03$ & $6.62 \mathrm{E}-04$ & 12.38 \\
0.2 & $1.04 \mathrm{E}-02$ & $9.41 \mathrm{E}-04$ & 9.01 \\
0.4 & $1.78 \mathrm{E}-02$ & $1.06 \mathrm{E}-03$ & 5.91 \\
0.6 & $2.40 \mathrm{E}-02$ & $1.45 \mathrm{E}-03$ & 6.04 \\
& & Total non-linear error $(\mathrm{CV})=15.60(\%)$ \\
\hline
\end{tabular}


(a)

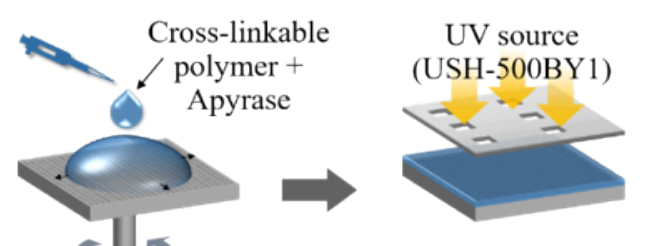

1. Spin coating

2. Photolithography

Apyrase-immobilized

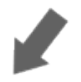
membrane

A smart biochip

(128 x 128 pixels)

3. Rinsing

(b)

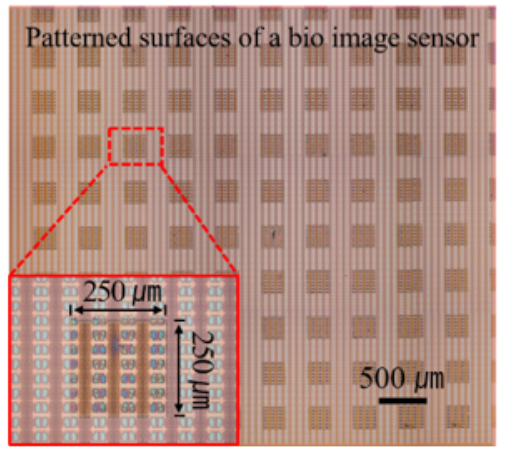

Figure 1. (a) Fabrication process for an apyrase -immobilized membrane. (b) Optical image of the surface of a completed bio-image sensor. The attempted size of a pattern is $250 \times 250 \mu \mathrm{m}^{2}$. 


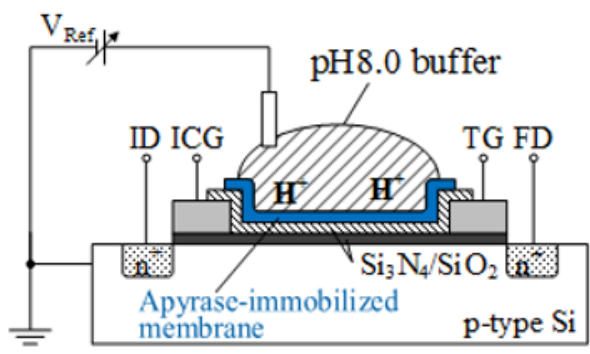

(a)

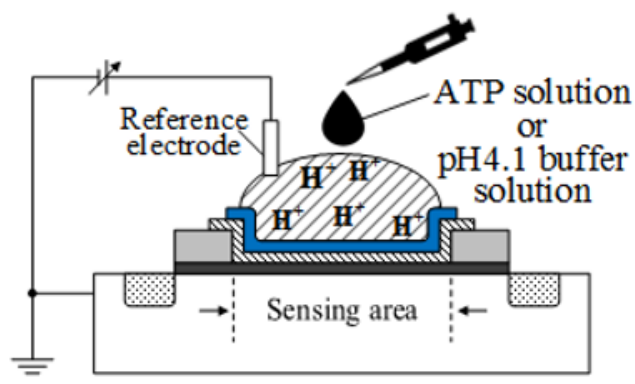

(a')

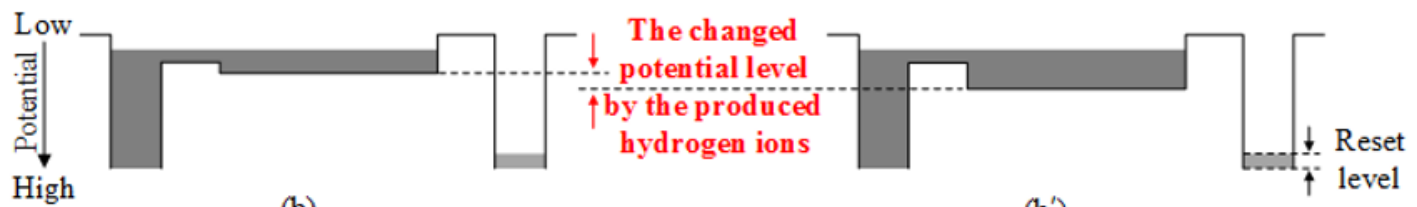

(b)

(b')

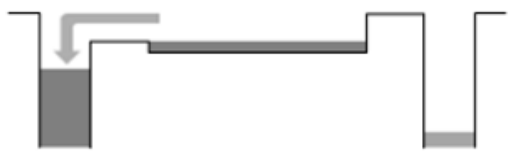

(c)

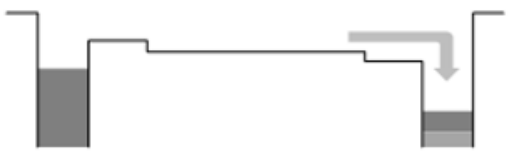

(d)

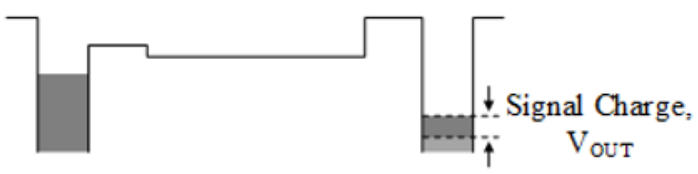

(e)

Figure 2. Schematic of the measurement principle using the charge transfer technique. (a) and (a') Cross-sectional images of an apyrase-immobilized pixel before and after dropping ATP solution or $\mathrm{pH}$ 4.1 buffer solution into $\mathrm{pH} 8.0$ buffer. (b) and (b') The changed potential levels by the amount of hydrogen ions. (c) Filling up the charge according to the potential level under the sensing area. (d) Charge transfer to FD. (e) Read-out signal charge into $V_{\text {OUT }}$. 


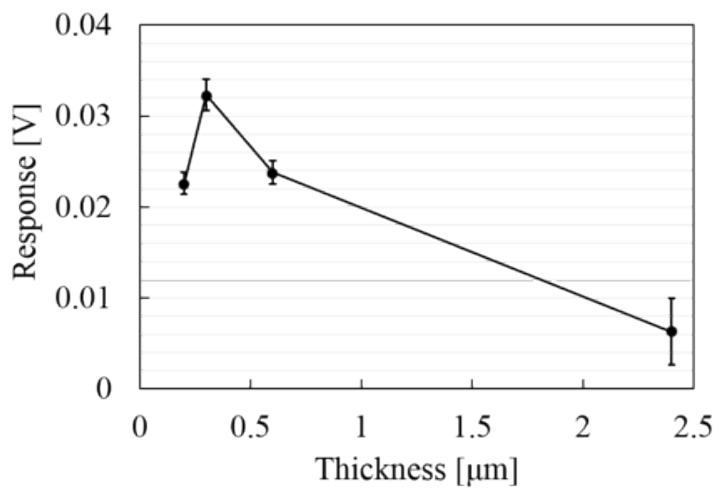

(a)

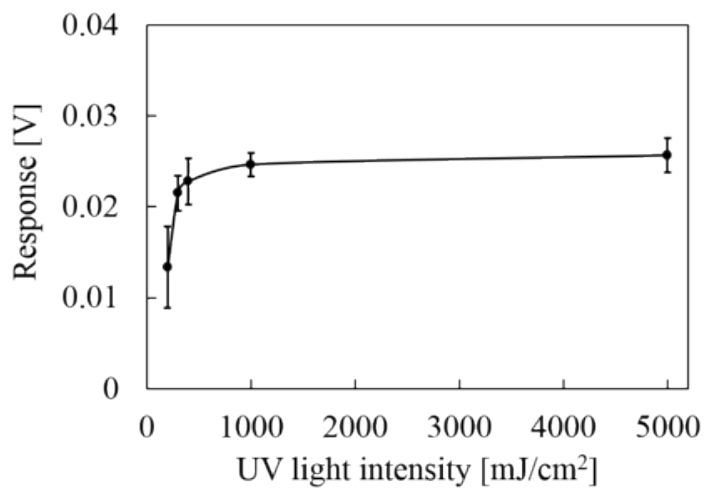

(b)

Figure 3. Response with respect to (a) the thickness of the apyrase-immobilized membrane $(\mathrm{n}=16384)$ and (b) the UV light power of lithographic method for patterning a membrane $(\mathrm{n}=36)$ at $1 \mathrm{mM}$ concentration ATP. 


\section{Optical image of the selected 12 pixels}

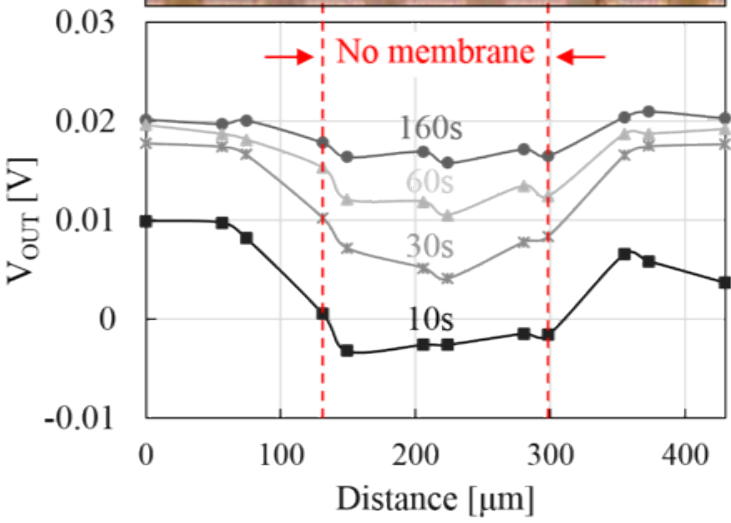

Figure 6. Diffusion characteristics of the produced $\mathrm{H}^{+}$ions at the apyraseimmobilized membrane are confirmed by the response change at sensing area without the membrane. The movement of $1 \mathrm{mM}$ ATP was measured sequentially by 12 pixels of the fabricated bio-image sensor. 


\section{Graphical Abstract}

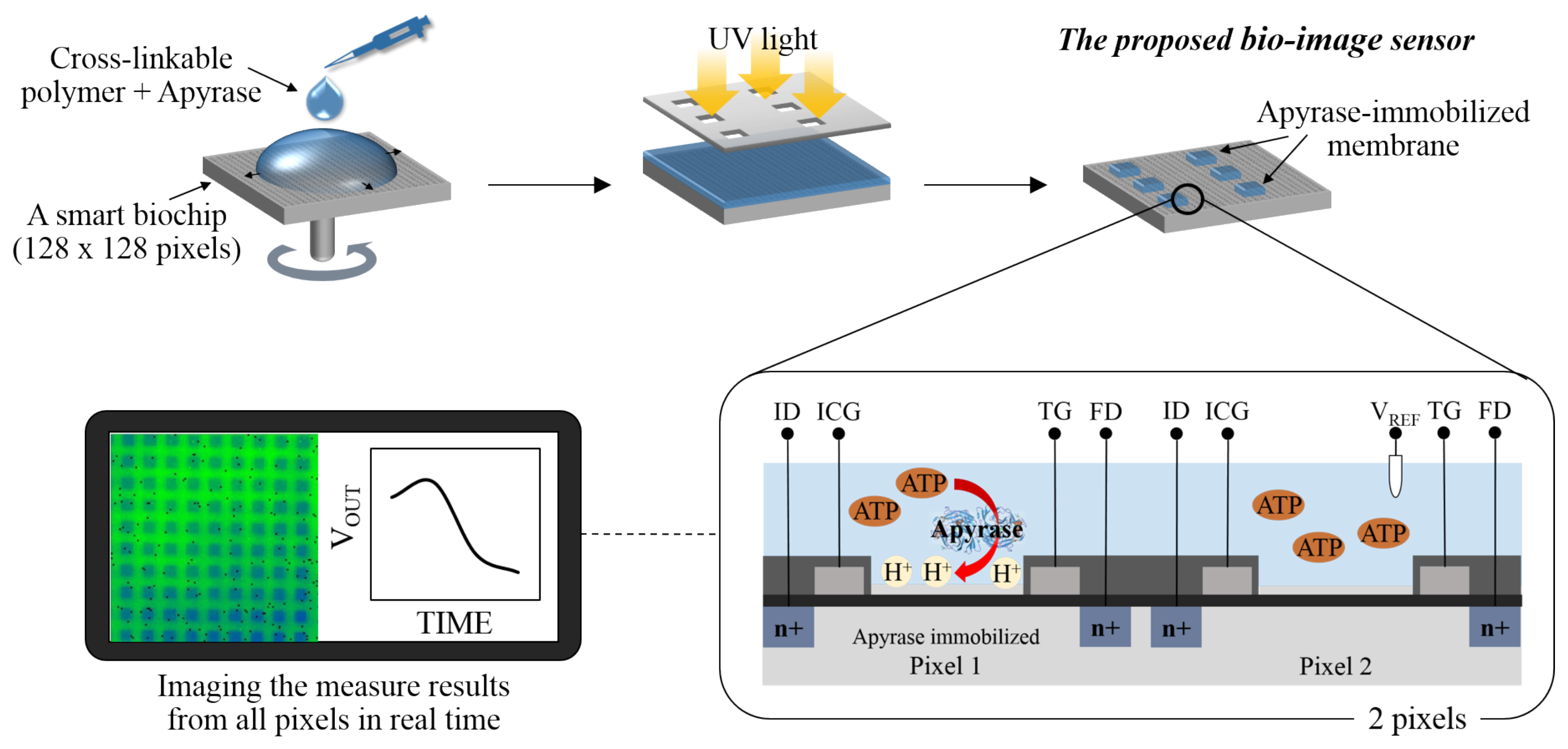

